\title{
Visibility of RF ablation lesions in native T1- weighted MRI reduces with time after ablation
}

\author{
Eugene Kholmovski ${ }^{1}{ }^{*}$, Ravi Ranjan ${ }^{2}$, Nathan Angel ${ }^{2}$, Sathya Vijayakumar ${ }^{2}$, Nassir F Marrouche ${ }^{2}$ \\ From 19th Annual SCMR Scientific Sessions \\ Los Angeles, CA, USA. 27-30 January 2016
}

\section{Background}

Cardiac RF ablation is a widely accepted procedure for treatment of ventricular tachycardia and atrial fibrillation. Late gadolinium enhanced MRI (LGE-MRI) can be used to assess RF ablation lesions. However, LGE-MRI requires contrast injection and the visibility of lesions and appearance considerably change with time after the injection. Recently, native (non-contrast) T1-weighted (T1w) MRI was proposed to visualize RF lesions immediately postablation. The main aim of this work was to study how the visibility and volume of RF lesions in native T1w MRI changes with time after ablation.

\section{Methods}

RF ablations of right and left ventricles of 2 canines were performed according to protocol approved by the local IACUC. RF lesions were created using Cool Flex catheter
(St. Jude Medical Inc.) at 30-40 Watts for 60 seconds. Imaging studies were performed on a 3T MRI scanner (Verio, Siemens HealthCare) at 0,2 , and 7 days post-ablation. Native T1w images of whole heart were acquired using 3D respiratory navigated, saturation recovery prepared GRE pulse sequence. Typical scan parameters were $\mathrm{TR} / \mathrm{TE}=3.1 /$ $1.4 \mathrm{~ms}$, flip angle of 10 degrees, $\mathrm{TI}=400 \mathrm{~ms}$, voxel size $=1.25 \times 1.25 \times 2.5 \mathrm{~mm}$. Fat saturation was applied immediately before data acquisition limited to $10 \%$ of RR interval.

Ablation lesions (enhanced regions) were manually segmented on native $\mathrm{T} 1 \mathrm{w}$ images acquired at different time point. Lesion volume was calculated for each ablation $(\mathrm{n}=9)$. Lesion volumes for 2 and 7 days post-ablation studies were normalized by the corresponding lesion volumes from acute (0 day) study. The visibility of ablation lesions in $\mathrm{T} 1 \mathrm{w}$ images was quantitatively assessed using Image

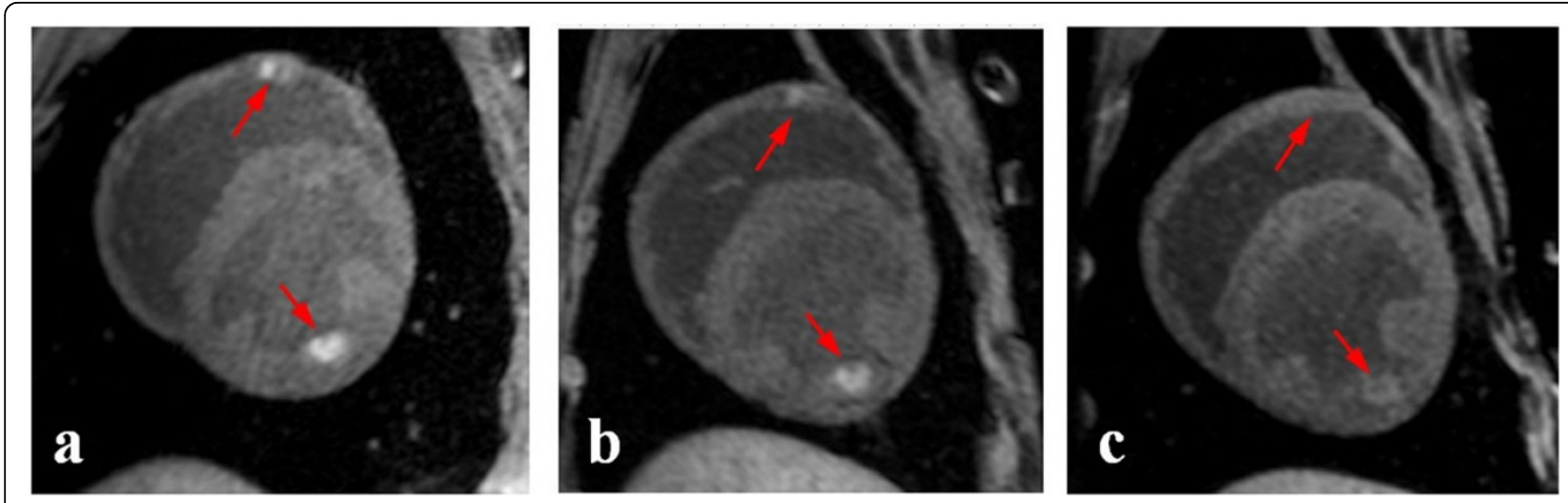

Figure 1 Representative T1w images of RF ablation lesions acquired (a) acutely, (b) 2 days post-ablation, and (c) 7 days post-ablation.

'UCAIR, Department of Radiology, University of Utah, Salt Lake City, UT, USA

Full list of author information is available at the end of the article 

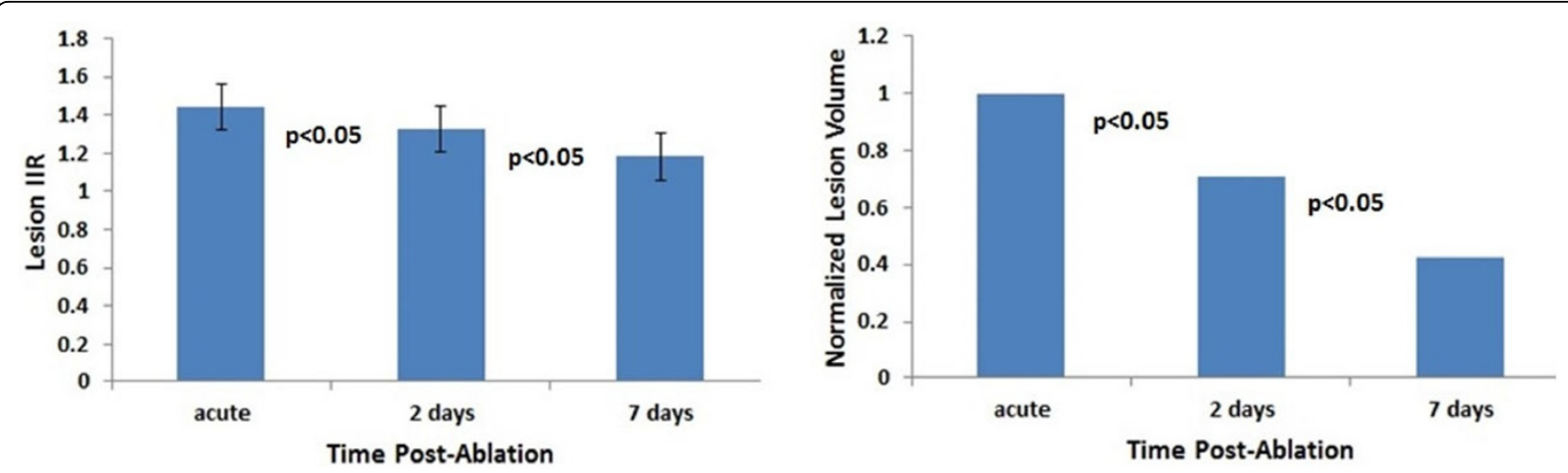

Figure 2 Left Panel - IIR of RF ablation lesions vs. time post-ablation. Right Panel - Normalized Lesion Volume vs. time post-ablation.

Intensity Ratio (IIR) metrics. IIR was calculated as a ratio of mean signal of lesion and mean signal of normal myocardium.

\section{Results}

Representative T1w images of RF ablation lesions acquired acutely, 2, and 7 days post-ablation are shown in Figure 1. These images clearly demonstrate that visibility of ablation lesions in native T1w images drastically reduces few days after ablation. Quantitate analysis shown that both normalized lesion volume and IIR, decrease significantly ( $\mathrm{p}<$ 0.05 ) as early as 2 days post-ablation (Figure 2). This trend continues further with time post-ablation, resulting in the inability to detect many ablation lesions on native $\mathrm{T} 1 \mathrm{w}$ images acquired 7 days post-ablation.

\section{Conclusions}

Reduction of $\mathrm{T} 1$ relaxation time of RF ablated myocardium has transient nature. The visibility of RF ablation lesions and volume of the corresponding enhanced regions in native T1w MRI drastically reduce with time after ablation. Non-contrast T1w MRI should be performed earlier ( $<2$ days) after RF ablation to achieve high contrast between ablated and normal myocardium and get an accurate estimate of lesion dimensions.

\section{Authors' details}

'UCAIR, Department of Radiology, University of Utah, Salt Lake City, UT, USA. ${ }^{2}$ CARMA Center, University of Utah, Salt Lake City, UT, USA.

Published: 27 January 2016

doi:10.1186/1532-429X-18-S1-P196

Cite this article as: Kholmovski et al:: Visibility of RF ablation lesions in native T1-weighted MRI reduces with time after ablation. Journal of Cardiovascular Magnetic Resonance 2016 18(Suppl 1):P196.
Submit your next manuscript to BioMed Central and take full advantage of:

- Convenient online submission

- Thorough peer review

- No space constraints or color figure charges

- Immediate publication on acceptance

- Inclusion in PubMed, CAS, Scopus and Google Scholar

- Research which is freely available for redistribution

Submit your manuscript at www.biomedcentral.com/submit 